\title{
False Belief Understanding in Children: The Comparison between Explanation Condition and Prediction Condition
}

\author{
Youqing CHEN \& Kaihong SUN \\ Institute of Applied Psychology, Hohai University, Nanjing, P. R. China
}

\begin{abstract}
To explore the performance of children in the two kinds of conditions: explanation condition and prediction condition, this study investigated the difference of the two conditions by the unexpected location task. The conclusions are as follows: Firstly, the grades that the children in 57-59-month-old get on the question that whether they can explain the reasons correctly in prediction condition are superior to the grades that they get in explanation condition. Secondly, specific reasons of the subjects differ in distinct conditions. The content of concrete wrong answers mainly concentrates on the two kinds of answers: the realistic location or have been moved and the need of desire of the protagonist.
\end{abstract}

KEYWORD: Theory of Mind; False Belief; Explanation Condition; Prediction Condition

\section{INTRODUCTION}

Premack and Woodruff firstly investigated the question that whether chimpanzees had the theory of mind in 1978. After that many psychologists explored the question. At the same time the study about the theory of mind had become a brand-new perspective and paradigm to explore children's understanding of mental representation (Li, F.Q. \& Gui, S.C., 2009; Liu, J. \& Jia, L.X., 2010). Having good theory of mind will greatly promote the development of the social cognitive ability, which can make children perceive themselves and others' mental state better in the process of communicating with people, and make appropriate response, eventually promoting them adapt to society better (Wang, Y.W. \& Zhang, Y.X., 2002). After decades of study, the focus of the study of theory of mind had certain change. Psychologists initially focused on the key age, and then focused on the influencing factors of theory of mind, and on the model of theory of mind and also probed into the internal mechanism. But throughout the past research, most scholars studied the predictive ability of children, and the ability of the explanation for children was less studied, The ability of the prediction and explanation is different. Letting children explain false belief behavior can be used to examine children's false belief reasoning, therefore studying the ability to explanation helps to further understand the development of theory of mind. Wimmer, $\mathrm{H}$. and Mayringer, H. (1998) firstly did the research in this field, and in the domestic, Zheng, H.L. (2006) found that development of recognizing the false belief and the ability of using the false belief to reasoning was not synchronous. So for the domestic children what are the differences and relations in the false belief understanding tasks between the two conditions: explanation condition and prediction condition is less involved. Therefore, this study presented the unexpected location task in the way of video files to compare the two different conditions, and to investigate the differences and the contacts of the ability of the prediction and explanation in the false belief task. This study is used to enrich children's theory of mind.

\section{METHODS}

\subsection{The subjects of study}

From Changzhou, Suzhou city, this study randomly selected 131 children in two kindergarten as the subjects, of which 29 children unfinished experiments, effective subjects were 102.The number of girl were 49, the number of boy were 53 . The age range of the participants was 42 months to 59 months, average months were 50.28 and the standard deviation was 4.88 .

\subsection{The experimental materials}

Therefore, this study presented the unexpected location task in the way of video files to measure 
children's theory of mind, there were two kinds of questions, one kind was the prediction condition, the other was the explanation condition, and the conditions of two kinds of questions were presented in the form of video. The production process of the video was as followers: firstly made the plot into Flash animation, and then increased the sound into the Flash by making use of the software Camtasia Studio.

The video story in the predicting condition: a sheep named Lanyangyang was playing with the car in the room. For a while, the other sheep named Meiyangyang called him to play out the room. And then he put the car in the toy box, they went out to play. When Lanyangyang was not at home, the sheep called Xiyangyang came, he went in the room, put the car in the quilt. After playing for a while Lanyangyang came back, he wanted to play with the car. Problems which were used to ask the children included memory control problem, the detection problem, idea problem, behavior prediction problem and explanation problem. The first two problems were used to examine if the children understand the story, if he or she gave the wrong answer, told the story again (up to three times).

The video story in the explanation condition was on the basis of video in the prediction of conditions increasing the follow plot: Lanyangyang came back. $\mathrm{He}$ directly went to the toy box to find his car. Problems included memory control problem, the detection problem and explanation problem about place. The first two problems were used to examine if the children understand the story, if he or she gave the wrong answer, told the story again (up to three times).

The contents of the explanation could be correct or wrong. For children using the wrong belief or before site to explain was correct. Wrong explanation can be divided into three types of codes. At the end of the experiment, the experimenter and an undergraduate student majored applied psychology who didn't know the purpose of major $\mathrm{s}$ classified the answers, and then analyzed the results of the two raters, the results show that the classification of high consistency $(\mathrm{K}=0.906)$.

\subsection{Procedure}

\subsubsection{The experimenter}

Two applied psychology undergraduates did the experiments. The participant did the tests one by one.

\subsubsection{The procedure}

(1) Preliminary experiments: firstly choosing four subjects and then the experimenters communicated with them to establish a good relationship with the children. After that the experiments said the instructions vividly and determined the children whether they were ready to start the experiment. The experimenters should record the data. All the performance during the process of experiment should be paid attention. The researcher analyzed the preparatory experiment data, pointed out the deficiencies in design, and corrected them.

(2) Formal experiments: Firstly the experimenter selected the appropriate experimental environment, established a good relationship with subjects, said the instructions and recorded data in time, and gave the child the reward finally.

\section{RESULTS}

\subsection{Right - wrong reasons}

To analyze whether grades have significant differences on the issue of content, the study did single dependent variable factor analysis of variance in which the condition of question, age variable were the independent variable, and the contents of explanation were the dependent variable. The results showed that the questions conditions had the main effect of significant, in more detail $F(1,101)=6.18$, $p<0.05$. Besides age classification had the main effect of significant, in more detail $F(5,101)=4.56, p<$ 0.05.Also the question condition and age classification had the significant interaction effect, in more detail $F(1,101)=4.49, p<0.05)$.

The study did the simple effect analysis of further inspection. The age of this research was divided into six classes, so doing independent sample t-test based on the six categories. The results showed that only on this age 57 to 59 , the results existed significant differences, the grades under the condition of predicting were better than the grades under the condition of explanation. The performances were shown in table 1.Analyzing the classification of age found that only the two classifications: 51 to 53 and 57 to 59 had significant differences in reasons, no significant difference between the other classifications of age.

Table 1. The independent t-test about the reasons under the two different conditions.

\begin{tabular}{|c|c|c|c|}
\hline months & mean difference & $t$ & $p$ \\
\hline $42-44$ & -0.17 & -0.68 & 0.52 \\
\hline $45-47$ & -0.01 & -0.15 & 0.88 \\
\hline $48-50$ & 0.03 & 0.16 & 0.88 \\
\hline $51-53$ & -0.08 & -0.73 & 0.48 \\
\hline $54-56$ & 0.14 & 0.83 & 0.42 \\
\hline $57-59$ & -0.92 & -5.61 & 0.00 \\
\hline
\end{tabular}

\subsection{Specific reasons}

The study found that although the correct ratio of the two different kinds of conditions about the questions was small, the correct ratio on the condition of 
prediction on the explanation problem about place was higher than that under the condition of explanation. Among the questions, the ratio of the kind of answer that the current place or the car had been transferred was the highest, which in the prediction condition, the ratio of this kind of question was $82.6 \%$ which was higher than $50 \%$. And the ratio of the kind of answer that no answers or not relevant answers were the lowest. But we also found that under the condition of prediction questions, little people answered the question using the reason of objective need or desire, this was a strange phenomenon.

Because of the analysis above was just a preliminary one. The analysis was about the different performances under the two kinds of conditions of different questions. Therefore further investigation with chi-square subjects under the conditions of different questions on specific reasons was needed. The study used the chi-square to analyze the specific reasons under the different conditions. The results showed that there were significant differences between them, in detail $F(3,102)=30.36, p<0.05$. The study used the chi-square to analyze the different performance on the concrete reasons under the two kings of conditions each other. The results found that four groups had the significant difference each other. The performances were shown in table 2 .

Table 2. The analysis of chi-square about the specific reasons under the different conditions.

\begin{tabular}{|c|c|c|c|}
\hline & correct & current place or been transferred & the need or desire \\
\hline current place or been transferred & 0.11 & & \\
\hline the need or desire & $15.55^{* *}$ & $25.05^{* *}$ & \\
\hline no or not relevant answers & $4.86^{*}$ & $8.69^{* *}$ & 2.41 \\
\hline
\end{tabular}

Note: $*$ indicates the significant level of 0.05 , general significant difference; $* *$ indicates the significant level of 0.01 , a very significant difference

\section{ANALYSIS AND DISCUSSION}

\subsection{The analysis of right-wrong reasons}

These children's explanation was divided into two parts considering its earlier research results and combining a number of researchers for the classification about the reasons: the right answers and the wrong answers. At the same time, the error explanation subdivided into three categories: current place or been transferred; expressing the purpose need or desire and giving no answers or not relevant answers.

The study found that question condition and age classification had the interactive effect, after the detailed analysis found that only on 57 to 59 months of age, the grades under the condition of prediction was superior to that under the condition of explanation. Existing research shows that 4 years old is the key age children receive theory of mind, but this was mainly in western countries and China's first-tier cities children as subjects of research results, and in the second-tier cities children as the subjects of study found that the key age of children receive theory of mind in our country were later than 4 years old (Zhang, T. et al, 2009). And the city that the two kindergartens located in are not first-tier cities, so according to the previous studies had shown that the key age of children receive theory of mind in our country were later than 4 years old. Chen, Y.Q. (2006) also found that most of the children aged 5 could correct understanding of false beliefs of others. So before 4 years old children couldn't well understand the false belief and at that age children couldn't understand false beliefs of others. Therefore children couldn't use the false belief to do deeper explain reasoning, so there was no significant difference on the ability that they explained and predicted the performance of the two kinds of conditions. In 57 to 59 months of age, because children at this stage is nearly five years old, so this stage children understand false belief to a certain extent, but due to explain conditions require children to explain the behavior of the protagonist, which in turn requires children have higher explanation reasoning ability, and the children of this stage haven't better development, so eventually the grades of the children in nearly five years old at this stage in condition of prediction are better that that under the condition of explanation.

\subsection{The analysis of specific reasons}

And on the specific reasons the research results showed that under the condition of prediction correct interpretation ratio $(15.22 \%)$ was higher than the number of interpretation under the condition of explanation (8.93\%), and further analysis showed that under the condition of different subjects in the concrete reasons existed significant differences in performance. We found that no children using the reasons that expressing the need or the desire to answer the question under the condition of prediction. This may led to the explanation between the need or desire and correct interpretation and the current place or been transferred and the need or desire had significant differences under different conditions. But the general research showed that more children can take the need or desire to do 
answer, so this was a different part compared the study of the Wimmer, H. and Mayringer, H. (1998) and Zheng, H.L. (2003) research, and this problem remains to be further explored.

Due to the correct explanation to some extent, means that the subjects could not only grasp the theory of mind of others, but also can do the casual inference on the basis of the behavior of others which also means that the subjects received theory of mind. The children whom used the current place or have been transferred to answer the questions did not really understand false belief but the answer they gave had a connection with the question. This indicated that they understood the problem to a certain extent. Instead there was no answer or not relevant answer was likely to mean that the meaning of the subjects did not understand the problem, so gave the irrelevant answers. Therefore this may led to the explanation between the no answers or not relevant answers and correct interpretation and the current place or been transferred and the no answers or not relevant answers had significant differences.

Comprehensive analysis of the reasons could initially forecasts the performances under the condition of prediction better than that under the condition of explanation for children. But the result was different from what the Wimmer, $\mathrm{H}$. and Mayringer, H. acquired (1998). They thought that the difficulty of explanation was not lower than the difficulty of the prediction, and may even harder than the difficulty of the prediction. But the study was based on western children as subjects, while research has suggested that theory of mind to a certain degree of consistency across different cultures, but the existing studies have shown that (Sui, X.S. \& Su, Y.J., 2003), some social factors influenced the development of the theory of mind such as psychological culture, living environment and other social factors. The study also found that children had the number of brothers and sisters and their relationship had a larger impact on the development of children's theory of mind. The western countries are most families with many children, but the situation in our country was relatively few. So it may be lead to the inconsistent performance for the children under the two kinds of culture environment.

This study only investigated the different performances of children's false belief under the two kinds of condition: the condition of prediction and the condition of explanation. The study involved less information under the condition of explanation. The study found that the passing rate children under the age of 3, 4 was not high, so the further experiments can by adding high age group, to increase the amount of information of the subjects. This study did not do further analysis the kind of wrong answer: not giving answers or not relevant answers. In the process of analyzing, the research found that some children answered the questions with traits of memories, some of the answer was a simple story, what was the reason for such answer, it was worth to explore. So in the later studying wrong answers could be subdivided, so as to further understand the development of the false belief understanding.

\section{CONCLUSIONS}

By the unexpected location task, this study examined the two kinds of performance difference of the children under the condition of explanation and prediction, the conclusions are as follows:

(1) The grades that the children in 57-59-monthold get on the question that whether they can explain the reasons correctly in prediction condition are superior to the grades that they get in explanation condition.

(2) Secondly specific reasons of the subjects differ in distinct conditions. The content of concrete wrong answers mainly concentrates on the two kinds of answers: the realistic location or have been moved and the need of desire of the protagonist

\section{REFERENCES}

[1] Chen, Y.Q. 2006. The Development Characteristics of Preschoolers' Theory of Mind in Different Tasks. Studies of Psychology and Behavior 4(4): 277-284.

[2] Li, F.Q. 2009. The Status and Development of the Children's Theory of Mind. Journal of Taiyuan University (Social Science Edition 8(1): 17-19.

[3] Liu, J. \& Jia, L.X. 2010. Present Conditions and Development Tendency of "Children's Theory of Mind". Journal of Mianyang Normal University 29(7): 139-142.

[4] Sui, X.S. \& Su, Y.J. 2003. Verifying the Cognitive Model of Theory of Mind. Acta Psychological Sinica 35(1): 5662.

[5] Wang, Y.W. \& Zhang, Y.X. 2002. Development of Theory of Mind of 3-6 Years Old Children. Psychological Development and Education (1): 11-14.

[6] Wimmer, H., \& Mayringer, H. 1998. False Belief Understanding in Young Children: Explanations do not Develop before Predictions. International of Behavioral Development 22(2): 403-422.

[7] Zhang, T. et al. 2009. The Development of 3 6 years old Children's Belief Reasoning. Psychological Development and Education (4): 15 -20.

[8] Zheng, H.L. 2006. The Research of $3 \sim 5$ years old children's explanation of false-belief-based action. Southwest University. 